\author{
O.P. Kogut*, P.I. Kogut**, T.N. Rudyanova*** \\ *Institute of Applied System Analysis, National Technical University - KPI \\ ** Oles Gonchar Dnipropetrovsk National University \\ ***Dnipropetrovsk State Finance Academy
}

\title{
A NOTE ON H-CONVERGENCE
}

\begin{abstract}
Вивчаються властивості Н-збіжності для послідовності рівномірно обмежених квадратних матриць $\left\{A_{\varepsilon} \in L^{\infty}\left(D ; R^{n \times n}\right)\right\}_{\mathcal{E}>0}$. Отримано достатні умови, які гарантують збіг Н-границі 3 * слаб̄кою границею в $L^{\infty}\left(D ; R^{n \times n}\right)$ таких послідовностей.
\end{abstract}

Introduction. The aim of this paper is to discuss some additional properties of the Hconvergence, which plays the key role in the homogenization theory of boundary value problems. So, the main object of our consideration is a sequence $\left\{A_{\mathcal{E}}(x)=\left[a_{i j}(x)\right]\right\}_{\mathcal{E}>0}$ of the uniformiy coercive and bounded matrices $A_{\varepsilon} \in L^{\infty}\left(\Omega ; R^{n \times n}\right)$. We suppose that this sequence is compact with respect to the $H$-convergence (see definition given belove). Let $A_{0} \in L^{\infty}\left(\Omega ; R^{n \times n}\right)$ be its $H$-limit. Since the sequence $\left\{A_{\mathcal{E}}(x)\right\}_{\varepsilon>0}$ is uniformly bounded in $L^{\infty}\left(\Omega ; R^{n \times n}\right)$, by Banach-Alaoglu Theorem it follows that there exists a matrix $A^{*} \in L^{\infty}\left(\Omega ; R^{n \times n}\right)$ such that (up to a subsequence) $A_{\varepsilon} \stackrel{w}{\longrightarrow} A^{*}$ weakly-* in $L^{\infty}\left(\Omega ; R^{n \times n}\right)$.

The question is to find conditions which would guarantee the equality $A_{0}=A^{*}$ almost everywhere in $\Omega$. Note that the answer is obvious in the case when

$$
A_{\varepsilon} \in L^{\infty}\left(\Omega ; R^{n \times n}\right) \cap W^{1,2}\left(\Omega, R^{n \times n}\right) \text { for every } \varepsilon>0
$$

and the sequence $\left\{A_{\mathcal{E}}(x)\right\}_{\varepsilon>0}$ is uniformly bounded in the Sobolev space $W^{1,2}\left(\Omega, R^{n \times n}\right)$. However, in general, the equality $A_{0}=A^{*}$ may be failed. This is the main reason why the optimal control problems in coefficients and shape optimization problems for systems governed by partial differential equations have no solutions in general (see, for instance, $[1$; $3 ; 7-9])$.

1. Notation and Preliminaries. Throughout the paper $\Omega$ is abounded open subset of $R^{n}$ with $n \geq 1$. The space $D^{\prime}(\Omega)$ of distributions in $\Omega$ is the dual of the space $C_{0}^{\infty}(\Omega)$. For two real numbers $1<p<+\infty, 1<q<+\infty$ such that $1 / p+1 / q=1$, the space $W_{0}^{1, p}(\Omega)$ is the

${ }^{\circ}$ O.P. Kogut, P.I. Kogut, T.N. Rudyanova, 2009 
closure of $C_{0}^{\infty}(\Omega)$ in the Sobolev space $W_{0}^{1, p}(\Omega)$, while $W_{0}^{-1, q}(\Omega)$ is the space of all distributions of the form $f=f_{0}+\sum_{j} D_{j} f_{j}$, with $f_{0}, f_{1}, \ldots, f_{n} \in L^{q}(\Omega)$ (i.e. $W_{0}^{-1, q}(\Omega)$ is the dual space of $W_{0}^{1, p}(\Omega)$. For any vector field $\vec{v} \in L^{p}(\Omega)=L^{p}\left(\Omega, R^{n}\right)$, the divergence is an element of the space $W_{0}^{-1, q}(\Omega)$ defined by the formula

$$
\left\langle\operatorname{div} \bar{\sigma}, \varphi_{W^{-1, q}(\Omega) W_{0}^{1, p}(\Omega)}=-\oint_{\Omega}(\bar{\sigma}, D \varphi)_{R^{n}} d x, \forall \varphi \in W_{0}^{1, p}(\Omega),\right.
$$

where $\langle\cdot, \cdot\rangle_{W^{-1, q}(\Omega) ; W_{0}^{1, p}(\Omega)}$ denotes the duality pairing between $W_{0}^{-1, q}(\Omega)$ and $W_{0}^{1, p}(\Omega)$ and $\langle\cdot, \cdot\rangle_{R^{n}}$ denotes the scalar product of two vectors in $R^{n}$.

A vector field $\vec{v}$ is said to be solenoidal, if $\operatorname{div} \vec{v}=0$. For any vector field $\vec{v} \in L^{p}(\Omega)$ the relations

$$
\begin{array}{r}
\langle\text { curl } \vec{v}, \varphi\rangle_{W^{-3, q}(\Omega), W_{0}^{1, p}(\Omega)}^{i j}=-\oint_{\Omega}\left(\nu_{i}, D_{j} \varphi-v_{j}, D_{i} \varphi\right) d x, \\
\quad \forall \varphi \in W_{0}^{1, p}(\Omega), \quad i, j=1, \ldots, n,
\end{array}
$$

define a skew-symmetric matrix curl $\overrightarrow{0}$, whose elements belong to the space $W^{-1, q}(\Omega) . A$ vector field $\vec{v}$ is said to be vortex-free, if $\operatorname{curl} \vec{v}=0$. We say that a vector field $\vec{v} \in L^{p}(\Omega)$ is potential, if $\vec{v}$ can be represented in the form $\vec{v}=D u$, where $u \in W^{1, p}(\Omega)$. Obviously, any potential vector is vortex-free. In the case when a vector field $\vec{v}$ smooth, the divergence div $\vec{v}$ and the curl $\vec{v}$ are defined as usual, that is,

$$
\operatorname{div} \vec{v}=\sum_{i=1}^{n} \frac{\partial v_{i}}{\partial x_{i}}, \quad(\operatorname{curl} \vec{v})_{i j}=\frac{\partial v_{i}}{\partial x_{j}}-\frac{\partial v_{j}}{\partial x_{i}} .
$$

We will also need to take the divergence and the curl of matrices, and then the above definitions can be applied to the row vectors, i.e., to the last index. Thus, with $M=\left(M_{i j}\right)$ and $P=\left(P_{i j}\right)$, we have

$$
(\operatorname{div} P)_{i}=\sum_{j=1}^{n} \frac{\partial P_{i j}}{\partial x_{j}}, \quad(\text { curl } M)_{i j k}=\frac{\partial M_{i j}}{\partial x_{k}}-\frac{\partial M_{i k}}{\partial x_{j}}
$$


Let us fix two constants $\alpha$ and $\beta$ such that $0<\alpha \leq \beta<+\infty$. We define $M_{\alpha}^{\beta}(\Omega)$ as a set of all matrices $A=\left[a_{i j}\right]$ in $L^{\infty}\left(\Omega ; R^{n \times n}\right)$ such that

$$
A(x) \geq \alpha I,(A(x))^{-1} \geq \beta^{-1} I, \text { a.e. in } \Omega \text {. }
$$

In (2.2) $I$ is the identity matrix in $R^{n \times n}$, and the above inequalities are in the sense of the quadratic forms defined by $(A \xi, \xi)_{R^{n}}$ for $\xi \in R^{n}$. Note that (2.2) implies the inequality

$$
|A(x)| \leq \beta \text { a.c. in } \Omega
$$

and that necessarily $\alpha \leq \beta$.

Throughout the paper $\varepsilon$ denotes a small parameter which varies in a strictly decreasing sequence of positive numbers converging to 0 . When we write $\varepsilon>0$, we consider only the elements of this sequence, while when we write $\varepsilon \geq 0$ we also consider its limit $\varepsilon=0$.

2. The definition of $\mathrm{H}$-convergence. In this section we recall the notion of $\mathrm{H}$ convergence introduced by Luc Tartar in 1977 (see[11]) and later developed by Francois Murat and L. Tartar (see [10]). Let $\left\{A_{\varepsilon}\right\}_{\varepsilon>0}$ be a sequence of matrices in $M_{\alpha}^{\beta}(\Omega)$ which are not necessarily symmetric.

DEFINTION 3.1. A sequence $\left\{A_{\varepsilon}\right\}_{\varepsilon>0}$ in $M_{\alpha}^{\beta}(\Omega)$ is said to be H-convergent to $A \in M_{\alpha}^{\beta}(\Omega)$ (in the symbol, $A_{\varepsilon} \stackrel{H}{\longrightarrow} A$ ) as $\varepsilon \rightarrow \infty$, if the following condition holds. Whenever vector fields $\vec{d}_{\varepsilon}, \vec{d}, \vec{v}_{\varepsilon}, \vec{v} \in L^{2}(\Omega)$ satisfy

$$
\begin{aligned}
& \vec{d}_{\varepsilon}=A_{\varepsilon}, \vec{v}_{\varepsilon}, \quad \forall \varepsilon>0, \\
& \vec{d}_{\varepsilon} \rightarrow \vec{d} \text { weakly in } L^{2}(\Omega), \\
& \vec{v}_{\varepsilon} \rightarrow \vec{v} \text { weakly in } L^{2}(\Omega), \\
&\left\{\text { div } \vec{d}_{\varepsilon}\right\}_{\varepsilon>0} \text { is relatively compact in } W^{-1,2}(\Omega), \\
&\left\{\text { curl } \vec{v}_{\varepsilon}\right\}_{\varepsilon>0} \text { is relatively compact in } W^{-1,2}\left(\Omega ; R^{n \times n}\right),
\end{aligned}
$$

then

$$
\vec{d}=A \vec{v} .
$$

It can be proved that the above definition of $H$-convergence is equivalent to the slightly different definition given in [10], concerning the limit behavior of the elliptic equations governed by matrix $A_{\varepsilon}$. Namely,

DEFINITION 3.2. A sequence $\left\{A_{\varepsilon}\right\}_{\varepsilon>0}$ of matrices in $M_{\alpha}^{\beta}(\Omega) H$-converges to a matrix $A \in M_{\alpha}^{\beta}(\Omega)$ if for every $f \in W^{-1,2}(\Omega)$ the sequence $\left\{u_{\varepsilon}\right\}_{\varepsilon>0}$ of the solutions to the Dirichlet problems 


$$
\left\{\begin{array}{c}
u_{\varepsilon} \in W_{0}^{1,2}(\Omega) \\
-\operatorname{div}\left(A_{\varepsilon} D u_{\varepsilon}\right)=f \text { in } D^{\prime}(\Omega)
\end{array}\right.
$$

satisfies

$$
\begin{aligned}
u_{\varepsilon} & \rightarrow u \text { weakly in } W_{0}^{1,2}(\Omega), \\
A_{\varepsilon} D u_{\varepsilon} & \rightarrow A D u \text { weakly in } L^{2}\left(\Omega, R^{n}\right),
\end{aligned}
$$

where $u$ is the solution to the problem

$$
\left\{\begin{array}{c}
u \in W_{0}^{1,2}(\Omega) \\
-\operatorname{div}(A D u)=f \text { in } D^{\prime}(\Omega)
\end{array}\right.
$$

The principle features of $H$-convergence we may attribute to the following well-know properties:

1) the $H$-limit of $H$-converging sequence is unique;

2) the set $M_{\alpha}^{\beta}(\Omega)$ is sequentially compact with respect to the $H$-convergence;

3) $H$-convergence is stable under the transposition of the matrices;

4) if $\left\{A_{\varepsilon}\right\}_{\varepsilon>0},\left\{B_{\varepsilon}\right\}_{\varepsilon>0} \subset M_{\alpha}^{\beta}(\Omega), A_{\varepsilon} \stackrel{H}{\longrightarrow} A, B_{\varepsilon} \stackrel{H}{\longrightarrow} B$ and for some open set $U \subset \Omega$ one has $A_{\varepsilon}=B_{\varepsilon}$ in $U$ for every $\varepsilon>0$, then $A=B$ in $U$.

The natural question is what is the relation between $H$-limit of a bounded sequence $\left\{A_{\varepsilon}\right\}_{\varepsilon>0}$ and its weak-* limit in $L^{\infty}\left(\Omega ; R^{n \times n}\right)$. As follows from the classical example, presented in the next section, in general, these limits may be drastically different.

4. One-dimensional case. Let $\Omega=(0,1)$. Let $a \in L^{\infty}(\Omega)$ be a positive function such that

$$
0<\alpha \leq a(x) \leq \beta<+\infty \text { a.c. in } \Omega \text {. }
$$

Assume that $a$ is periodic with the period $T$. Set $a_{\varepsilon}=a(x / \varepsilon)$. Consider the particular case of the boundary value problem (6.3)

$$
\left\{\begin{array}{c}
-\frac{d}{d x}\left(a_{\varepsilon} \frac{d u_{\varepsilon}}{d x}\right)=f \text { in } \Omega \\
u_{\varepsilon}(0)=u_{\varepsilon}(1)=0 .
\end{array}\right.
$$

For each $\varepsilon>0$ this problem has a unique solution $u_{\varepsilon} \in W_{0}^{1,2}(\Omega)$. First of all we will show that the sequence $\left\{u_{\varepsilon}\right\}_{\varepsilon>0}$ has a limit in the weak topology of $u_{\varepsilon} \in W_{0}^{1,2}(\Omega)$. Indeed, as follows from (4.2), after multiplication by $u_{\varepsilon}$, integration by parts, and use of (4.1), one has

$$
\alpha\left\|\frac{d u_{\varepsilon}}{d x}\right\|_{L^{2}(\Omega)}^{2} \leq\|f\|_{L^{2}(\Omega)}\left\|u_{\varepsilon}\right\|_{L^{2}(\Omega)} .
$$


Hence

$$
\left\|u_{\varepsilon}\right\|_{L^{2}(\Omega)}+\left\|\frac{d u_{\varepsilon}}{d x}\right\|_{L^{2}(\Omega)} \leq \frac{C}{\alpha}\|f\|_{L^{2}(\Omega)},
$$

where the Poincare constant $C$ is independent of $\varepsilon$. Thus the sequence of solutions $\left\{u_{\varepsilon}\right\}_{\varepsilon>0}$ is relatively compact with respect to the weak topology of $W_{0}^{1,2}(\Omega)$. To identify its limit we note that from (4.1) and (4.3), it follows that there exists a subsequence, still denoted by $\varepsilon$, such that

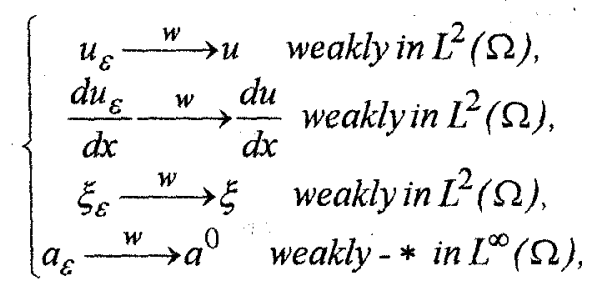

where $\xi_{\varepsilon}=a_{\varepsilon} d u_{\varepsilon} / d x, a^{0}$ is the mean value of $a$. Here we used the fact that by the periodicity of $a_{\varepsilon}$, we have

$$
a^{0}=\langle a\rangle:=\frac{1}{T} \int_{0}^{T} a(x) d x .
$$

Moreover, from (4.2) we derive that at the limit,

$$
\frac{d}{d x} \xi=f \text { in } \Omega \text {. }
$$

As was mentioned above, from (4.4) we have $u_{\varepsilon} \stackrel{w}{\longrightarrow} u$ weakly in $W_{0}^{1,2}(\Omega)$ which implies, by continuity of the trace operator, that $u(0)=u(1)=0$. Now we establish the relation between $\xi$ and $u$. Clearly, from (4.1)-(4.3), since $f \in L^{2}(\Omega)$, we have a priori estimate

$$
\left\|\xi_{E}\right\|_{L^{2}(\Omega)}+\left\|\frac{d \xi_{\varepsilon}}{d x}\right\|_{L^{2}(\Omega)} \leq C,
$$

where $C$ is independent of $\varepsilon$. Consequently, and using the Rellich-Kondrashov theorem, one has

$$
\xi_{\varepsilon} \stackrel{w}{\longrightarrow} \xi \text { weakly in } W_{0}^{1,2}(\Omega) \text {, hence strongly in } L^{2}(\Omega) .
$$

Notice that $\xi_{\varepsilon}$ is the product of two weakly converging quantities. In general, this does not imply that the limit $\xi$ is the product of the weak limits $a^{0}$ and $d u / d x$. To begin with, we remember that

$$
\frac{d u_{\mathcal{E}}}{d x}=\frac{1}{a_{\varepsilon}} \xi_{\mathcal{E}}
$$

where $0<\frac{1}{\beta} \leq \frac{1}{a_{\varepsilon}} \leq \frac{1}{a}<+\infty$, and so, by periodicity, 


$$
\frac{1}{a_{\varepsilon}} \stackrel{w}{\longrightarrow}\left(\frac{1}{a}\right):=\frac{1}{T} \int_{0}^{T} \frac{1}{a(x)} d x \text { weakly in } L^{\infty}(\Omega) .
$$

Since the term $\left(1 / a_{\mathcal{E}}\right) \xi_{\mathcal{E}}$ is the product of the weakly convergent quantity $1 / a_{\delta}$ by the strongly convergent one $\xi_{\varepsilon}$, its limit is the product of the limits of $1 / a_{\varepsilon}$ and $\xi_{\varepsilon}$. Thus

$$
\frac{1}{a_{\varepsilon}} \xi_{\varepsilon} \stackrel{w}{\longrightarrow}\left(\frac{1}{a}\right) \xi \text { weakly in } L^{2}(\Omega)
$$

Consequently, from (4.6), we have $\frac{d u}{d x}=\left(\frac{1}{a}\right) \xi$. Now making use of (16), it follows that $u$ is the solution of the limit boundary value problem

$$
\left\{\begin{array}{c}
-\left\langle\frac{1}{a}\right\rangle^{-1} \frac{d^{2} u}{d x^{2}}=f \text { in } \Omega, \\
u(0)=u(1)=0 .
\end{array}\right.
$$

This problem has a unique solution, so the whole sequence $\left\{u_{\varepsilon}\right\}_{\mathcal{E}>0}$ converges to $u$ weakly in $W_{0}^{1,2}(\Omega)$.

Thus, due to the Definition 3.2, we come to following conclusion:

$$
a_{E} \stackrel{H}{\longrightarrow}\left(\frac{1}{a}\right)^{-1}
$$

whereas $a_{\varepsilon} \stackrel{w}{\longrightarrow}\langle a\rangle$ weakly * in $L^{\infty}(\Omega)$. However, in general; $\left\langle\frac{1}{a}\right\rangle^{-1} \neq\langle a\rangle$, and we obtain the required.

5. The nonlinear Dirichlet problem. We begin with the more precise description of our main object. For a fixed matrix $A \in M_{\alpha}^{\beta}(D)$ and a real number $1 \leqslant p ; \leqslant+\infty$, we define the mapping $a: R^{n} \times R^{n} \mapsto R^{n}$ as follows

$$
a(x, \zeta)=A(x)|\zeta|^{p-2} \zeta
$$

Then (see, for instance, [2], [4]) there exist two constants $c_{0}, c_{1}$ with $0<c_{0} \leq c_{1}<+\infty$ such that, a.e. in $R^{n}$ and for every $\zeta_{1}, \zeta_{2} \in R^{n}$, we have

in the case $2 \leq p<+\infty$

$$
\begin{aligned}
& \left(a\left(x, \zeta_{1}\right)-a\left(x, \zeta_{2}\right), \zeta_{1}-\zeta_{2}\right)_{R^{n}} \geq c_{0}\left|\zeta_{1}-\zeta_{2}\right|_{R^{n}}^{p} \\
& \mid\left(a\left(x, \zeta_{1}\right)-\left.a\left(x, \zeta_{2}\right)\right|_{R^{n}} \leq c_{1}\left(\left|\zeta_{1}\right|_{R^{n}}+\left|\zeta_{2}\right|_{R^{n}}\right)^{p-2}\left|\zeta_{1}-\zeta_{2}\right|_{R^{n}}\right.
\end{aligned}
$$

in the case $1<p \leq 2$

$$
\begin{gathered}
\left(a\left(x, \zeta_{1}\right)-a\left(x, \zeta_{2}\right), \zeta_{1}-\zeta_{2}\right)_{R^{n}} \geq c_{0}\left(\left|\zeta_{1}\right|_{R^{n}}+\left|\zeta_{2}\right|_{R^{n}}\right)^{p-2}\left|\zeta_{1}-\zeta_{2}\right|_{R^{n}}^{2} \\
\mid\left(a\left(x, \zeta_{1}\right)-\left.a\left(x, \zeta_{2}\right)\right|_{R^{n}} \leq c_{1}\left|\zeta_{1}-\zeta_{2}\right|_{R^{n}}^{p-1}\right.
\end{gathered}
$$


In particular (5.1)-(5.4) imply that a.e. in $R^{n}$ and for any $\zeta \in R^{n}$ :

$$
(a(x, \zeta), \zeta)_{R^{n}} \geq c_{0}|\zeta|_{R^{n}}^{p}, \quad|a(x, \zeta)|_{R^{n}} \leq c_{1}|\zeta|_{R^{n}}^{p-1} .
$$

Since $A \in M_{\alpha}^{\beta}(D)$ it is easy to verify that (5.1)-(5.4) are satisfied with

$$
\begin{gathered}
c_{0}=2^{2-p} \alpha \text { and } c_{1}=(p-1) \beta \text { for } p \geq 2, \text { and } \\
c_{0}=\alpha, c_{1}=2^{2-p} \beta \text { for } p \leq 2 .
\end{gathered}
$$

As a result, for a given function $a_{0} \in L^{\infty}(D)$ such that $a_{0}(x) \geq 0$ a.e. in $D$, the operator

defined via the paring

$$
A y=-\operatorname{div}(a(x, D y))+a_{0}(x)|y|^{p-2} y
$$

$$
\begin{aligned}
\langle A y, v\rangle_{W^{-1, q}(\Omega), W_{0}^{1, p}(\Omega)} & =\int_{\Omega}\left(A(x)|D y|_{R^{n}}^{p-2} D y, D v\right)_{R^{n}} d x+ \\
& +\int_{\Omega} a_{0}(x)|y|^{p-2} y v d x \quad \forall y, v \in W_{0}^{1, p}(\Omega)
\end{aligned}
$$

turns out to be coercive, strictly monotone from $W_{0}^{1, p}(\Omega)$ into its dual $W_{0}^{-1, q}(\Omega)$, and demicontinuous in the following sense: $y_{k} \rightarrow y_{0}$ strongly in $W_{0}^{1, p}(\Omega)$ implies that $A_{y k} \rightarrow A_{y 0}$ weakly in $W_{0}^{-1, q}(\Omega)$ (see [5], [6]). Then by well-known existence results for nonlinear elliptic equations with strictly monotone demi-continuous cocrcive operators (see [5], [12]), one can easily see that for every open set $\Omega$ and every $f \in L^{q}(\Omega)$ the nonlinear Dirichlet boundary value problem

$$
A y=f \text { in } \Omega, y \in W_{0}^{1, p}(\Omega)
$$

has a unique weak solution in $W_{0}^{1,2}(\Omega)$. Let us recall that a function $y$ is the weak solution of (5.6) if

$$
\begin{gathered}
y \in W_{0}^{1, p}(\Omega) \\
\int_{\Omega}\left(A(x)|D y|_{R^{n}}^{p-2} D y, D v\right)_{R^{n}} d x+\int_{\Omega} a_{0}(x)|y|^{p-2} y v d x=\int_{\Omega} f v d x \quad \forall v \in W_{0}^{1, p}(\Omega) .
\end{gathered}
$$

Following [2], [4], we conclude this section by some results concerning the estimates for the solution of Dirichlet problem (5.6).

PROPOSITION 5.1. For every $A \in M_{\alpha}^{\beta}(D), a_{0} \in L^{\infty}(D)\left(a_{0}(x) \geq 0\right.$ a.e. in $\left.D\right)$, and $f \in W^{-1, q}(\Omega)$ the unique weak solution $y_{A}$ to the problem (5.6) satisfies the estimates:

$$
\int_{\Omega}\left|D y_{A}\right|_{R^{n}}^{p} d x \leq C\|f\|_{W^{-1, q}(\Omega)}^{q},\left\|y_{A}\right\|_{W_{0}^{1, p}(\Omega)} \leq C
$$

where $C$ is a constant depending only on $p, \alpha$, and $\beta$. 
6. The main result. In this section we introduce a class of matrices $A=\left\lfloor a_{i j}\right\rfloor$, as a subset of $M_{\alpha}^{\beta}(\Omega)$, for which the $H$-limit and weak-* limil in $L^{\infty}\left(\Omega ; R^{n \times n}\right)$ are the same. Let $\xi_{1}, \xi_{2}$ be given functions of $L^{\infty}(D)$ such that $0<\xi_{1}(x)<\xi_{2}(x)$ a. e. in $\Omega$. Let $\left\{Q_{1}, \ldots Q_{n}\right\}$ be a collection of nonempty compact subsets of $W^{-1, q}(\Omega)$. To define the class of admissible matrices, we introduce the following set

$$
U_{\text {sol }}=\left\{A=\left[a_{1}, \ldots a_{n}\right] \in M_{\alpha}^{\beta}(\Omega) \mid d i v a_{i} \in Q_{i}, \forall i=1, \ldots n\right\}
$$

assuming that $U_{\text {sol }} \subset L^{\infty}\left(\Omega ; R^{n \times n}\right)$ is a nonempty set.

DEFINITION 6.1. We say that a matrix $A=\left\lfloor a_{i j}\right\rfloor$ is admissible to the nonlinear Dirichlet problem (5.6) if $A \in U_{\text {sol }}$.

To begin with, we prove the following result:

PROPOSITION 6.2. The set $U_{\text {sol }}$ is sequentially compact with respect to the weak-* topology of $L^{\infty}\left(\Omega ; R^{n \times n}\right)$.

Proof. Let $\left\{A_{k}=\left[\vec{a}_{1 k}, \ldots \vec{a}_{n k}\right]_{k \in N} \subset U_{\text {sol }}\right.$ be an arbitrary sequence of admissible matrices. Since $U_{\text {sol }} \subset M_{\alpha}^{\beta}(\Omega)$ and $M_{\alpha}^{\beta}(\Omega)$ is the sequentially weakly-* compact subset of $L^{\infty}\left(\Omega ; R^{n \times n}\right)$, we may suppose that there exist a matrix $A_{0}=\left[\vec{a}_{10}, \ldots \vec{a}_{n 0}\right] \in M_{\alpha}^{\beta}(\Omega)$ and elements $f_{i} \in Q_{i}, i=1, \ldots n$ such that

$$
\int_{\Omega}\left(\vec{a}_{i k}, \phi\right)_{R^{n}} d x \stackrel{k \rightarrow \infty}{\longrightarrow} \int_{\Omega}\left(\vec{a}_{i 0}, \phi\right)_{R^{n}} d x, \forall \phi \in L^{1}(\Omega)=L^{1}\left(\Omega, R^{n}\right), \quad \forall i=1,2, \ldots n
$$

and

$$
d i v \vec{a}_{i k} \stackrel{k \rightarrow \infty}{\longrightarrow} f_{i} \text { in } W^{-1, q}(\Omega) \forall i=1,2, \ldots n .
$$

It remains to prove that

$$
\operatorname{div} \vec{a}_{i 0}=f_{i} \text { for all } i=1,2, \ldots n \text {. }
$$

To do this, we choose $\phi$ in (6.2) as a potential vector, that is, $\phi=D v$, where $v \in W_{0}^{1, p}(\Omega)$. Then, in view of (2.1), the relation (6.3) implies

$$
\begin{aligned}
\int_{\Omega}\left(\vec{a}_{i k}, D v\right)_{R^{n}} d x=-\left\langle d i v \vec{a}_{i k}, v\right\rangle_{W^{-1, q}(\Omega), W_{0}^{1, p}(\Omega)} & \stackrel{k \rightarrow \infty}{\longrightarrow}-\left\langle f_{i}, v\right\rangle_{W^{-1, q}(\Omega), W_{0}^{1, p}(\Omega)} \forall i=1, \ldots, n .
\end{aligned}
$$

Using this and relation (6.2),we finally get

$$
\begin{aligned}
& \lim _{k \rightarrow \infty} \int_{\Omega}\left(\vec{a}_{i k}, D v\right)_{R^{n}} d x=\int_{\Omega}\left(\vec{a}_{i 0}, D v\right)_{R^{n}} d x \\
& =-\left\langle\operatorname{div} \vec{a}_{i 0}, v\right\rangle_{W^{-1, q}(\Omega), W_{0}^{1, p}(\Omega)}=-\left\langle f_{i}, v\right\rangle_{W^{-1, q}(\Omega), W_{0}^{1, p}(\Omega)} \forall i=1, \ldots, n .
\end{aligned}
$$

As a result, we have $A_{0}=\left[\vec{a}_{10}, \ldots \vec{a}_{n 0}\right] \in U_{\text {sol }}$. This concludes the proof. 
In what follows, we make use of the following result (for the proof we refer to [13]).

LEMMA 6.3. Let $\left\{\vec{f}_{k}\right\}_{k \in N} \subset L^{q}(\Omega),\left\{q_{k}\right\}_{k \in N} \subset L^{p}(\Omega)$ be the bounded sequences of vector-functions such that

$$
\vec{f}_{k} \stackrel{w}{\longrightarrow} \vec{f}_{0} \text { in } L^{q}(\Omega) \text { and } \vec{g}_{k} \stackrel{w}{\longrightarrow} \vec{g}_{0} \text { in } L^{p}(\Omega) \text {. }
$$

If

$$
\left\{\text { div } f_{k}\right\}_{k \in N} \text { is compact with respect to the strong topology of } W^{-1, q}(\Omega),(6.4)
$$

and

$$
\operatorname{curl} \vec{g}_{k}=0 \quad \forall k \in N
$$

then

$$
\lim _{k \rightarrow \infty} \int_{\Omega} \phi\left(\vec{f}_{k}, \vec{g}_{k}\right)_{R^{n}} d x=\int_{\Omega} \phi\left(f_{0}, \vec{g}_{0}\right)_{R^{n}} d x, \forall \phi \in C_{0}^{\infty}(D)
$$

REMARK 6.4. The equality (6.6) can be interpreted as the weak-* convergence in $L^{l}(\Omega)$ of the sequence $\left\{\left(\vec{f}_{k}, g_{k}\right)_{R^{n}}\right\}_{k \in N}$ to the element $\left(\vec{f}_{0}, \vec{g}_{0}\right)_{R^{n}}$.

Let us denote by $\Xi \subset L^{\infty}\left(\Omega ; R^{n \times n}\right) \times W_{0}^{1, p}(\Omega)$ the set of all admissible pairs $\left(A, y_{A}\right)$ : matrix and the corresponding solution to the boundary value problem $(5.7)-(5.8)$. Now we are in a position to study the topological properties of this set. Let $\tau$ be the topology on the set $L^{\infty}\left(\Omega ; R^{n \times n}\right) \times W_{0}^{1, p}(\Omega)$ which we define as the product of the weak-* topology of $L^{\infty}\left(\Omega ; R^{n \times n}\right)$ and the weak topology of $W_{0}^{1, p}(\Omega)$.

THEOREM 6.5. For every $f \in W^{-1, q}(\Omega)$ the set $\Xi$ is sequentially $\tau$-closed.

Proof. Let $\left\{\left(A_{k}, y_{k}\right)\right\}_{k \in N} \subset \Xi$ be any $\tau$-convergent sequence. Let $\left(A_{0}, y_{0}\right)$ be its $\tau$ limit. Our aim is to prove that $\left(A_{0}, y_{0}\right) \in \Xi$. Let us set

$$
\begin{aligned}
\Im & (A, y)=-\operatorname{div}\left(A(x)|D y|_{R^{n}}^{p-2} D y\right)+a_{0}(x)|y|^{p-2} y=\Im_{1}(A, y)+\Im_{2}(A, y), \\
& \Im_{1}(A, y)=-\operatorname{div}\left(\left.A(x)|D y|\right|_{R^{n}} ^{p-2} D y\right)=-\operatorname{div} a(A(x), D y) .
\end{aligned}
$$

By Proposition 6.2 and the initial assumptions, we have $A_{0} \in U_{\text {sol }}$, therefore,

$$
\begin{aligned}
A_{k} \stackrel{w}{\longrightarrow} A_{0}=\left[\vec{a}_{10}, \ldots \vec{a}_{n 0}\right] \text { weakly-* in } L^{\infty}\left(\Omega ; R^{n \times n}\right), \\
\operatorname{div} \vec{a}_{i k} \rightarrow \operatorname{div} \vec{a}_{i 0} \text { strongly in } W^{-1, q}(\Omega), \forall i=1, \ldots n . \\
y_{k} \stackrel{w}{\longrightarrow} y_{0} \text { in } W_{0}^{1, p}(\Omega) .
\end{aligned}
$$

Hence

$$
\begin{gathered}
\left\{\left.D y_{k}\right|_{R^{n}} ^{p-2} D y_{k}\right\}_{k \in N} \text { is bounded in } L^{q}(\Omega), \quad q=p /(p-1), \\
\left\{\left.y_{k}\right|^{p-2} y_{k}\right\}_{k \in N} \text { is bounded in } L^{q}(\Omega),
\end{gathered}
$$




$$
y_{k} \rightarrow y_{0} \text { in } L^{p}(\Omega), \quad y_{k}(x) \rightarrow y_{0}(x) \text { a.e. in } \Omega .
$$

Then, by (6.11) and monotonicity of the function $g(\zeta)=|\zeta|^{p-2} \zeta$, we have

$$
\left|y_{k}\right|^{p-2} y_{k} \rightarrow\left|y_{0}\right|^{p-2} y_{0} \text { almost every wherein } \Omega \text {. }
$$

Using this and (6.10), we conclude (see[6])

$$
\left|y_{k}\right|^{p-2} y_{k} \stackrel{w}{\longrightarrow}\left|y_{0}\right|^{p-2} y_{0} \text { in } L^{q}(\Omega) \text {. }
$$

Consider the following sequence

$$
\left\{f_{k}:=f-a_{0}\left|y_{k}\right|^{p-2} y_{k}\right\}_{k \in N} .
$$

It is clear that $f_{k} \in W^{-1, q}(\Omega) \forall k \in N$ and since the embedding $L^{q}(\Omega) \subset W^{-1, q}(\Omega)$ is compact it follows that

$$
f_{k} \rightarrow f_{0}=f-a_{0}\left|y_{0}\right|^{p-2} y_{0} \text { strongly in } W^{-1, q}(\Omega) .
$$

In view of this and the fact that

$$
-\operatorname{div} a\left(A_{k}(x), D y_{k}\right)=f_{k} \text { in } \Omega, \forall k \in N,
$$

we come to the conclusion (see (5.1)-(5.5)): $\left\{a\left(A_{k}(x), D y_{k}\right\}_{k \in N}\right.$ is the bounded sequence in $L^{q}(\Omega)$. So, passing to a subsequence, we may assume that there exists a vector-function $\vec{\xi} \in L^{q}(\Omega)$ such that

$$
a\left(A_{k}(x), D y_{k}\right)=A_{k}\left|D y_{k}\right|_{R^{n}}^{p-2} D y_{k}=: \vec{\xi}_{k} \stackrel{w}{\longrightarrow} \vec{\xi} \text { in } L^{q}(\Omega) .
$$

As a result, the limit passage in the relation as $k \rightarrow \infty$ (in the sense of the distributions)

$$
-\operatorname{div} \vec{\xi}_{k}=\int-a_{0}\left|y_{k}\right|^{p-2}\left|y_{k}\right|, \text { in } D^{\prime}(\Omega)
$$

gets

$$
-d i v \vec{\xi}=f-a_{0}\left|y_{0}\right|^{p-2}\left|y_{0}\right|, \text { in } D^{\prime}(\Omega)
$$

It remains only to show that

$$
\vec{\xi}=A_{0}\left|D y_{0}\right|_{R^{n}}^{p-2} D y_{0}
$$

To do so, we consider the scalar function

$$
v(x)=(z, x)_{R^{n}},
$$

where $z$ is a fixed element of $R^{n}$. Since the operator $\Im_{1}$ is strictly monotone, it follows that for every $z \in R^{n}$ and every positive function $\phi \in C_{0}^{\infty}(\Omega)$, we have

$$
\int_{\Omega} \phi(x)\left(a\left(A_{k}, D y_{k}\right)-a\left(A_{k}, D v\right), D y_{k}-D v\right)_{R^{n}} d x \geq 0,
$$

or, taking into account $(6.15)$, this inequality can be rewritten as

$$
\int_{\Omega} \phi(x)\left(a\left(A_{k}, D y_{k}\right)-a\left(A_{k}, z\right), D y_{k}-z\right)_{R^{n}} d x \geq 0 .
$$

Our next intention is to pass to the limit in (6.16) as $k \rightarrow \infty$ using Lemma 6.3. Since 


$$
\left.\begin{array}{c}
-\operatorname{diva}\left(A_{k}, D y_{k}\right) \rightarrow f-a_{0}\left|y_{0}\right|^{p-2}\left|y_{0}\right| \text { strongly in } W^{-1, q}(\Omega), \\
\operatorname{curv}\left(D y_{k}-z\right)=\operatorname{curv} D y_{k}=0, \forall k \in N,
\end{array}\right\}
$$

it remains to show that the sequence $\left\{\operatorname{div} a\left(A_{k}, z\right)\right\}_{k \in N}$ is compact with respect to the strong topology of $W^{-1, q}(\Omega)$.

Indeed, for every $\phi \in C_{0}^{\infty}(\Omega)$, we have

$$
\begin{gathered}
\left\langle-\operatorname{diva}\left(A_{k}, z\right), \phi\right\rangle_{W^{-1, q}(\Omega) ; W_{0}^{1, p}(\Omega)}=\int_{\Omega}\left(a\left(A_{k}, z\right), D \phi\right)_{R^{n}} d x \\
=\int_{\Omega}\left(A_{k}(x)|z|_{R^{n}}^{p-2} z, D \phi\right) d x=|z|^{p-2} \int_{\Omega}\left(\left[\begin{array}{c}
\left(\vec{a}_{1 k}(x), z\right)_{R^{n}} \\
\cdots \\
\left(\vec{a}_{n k}(x), z\right)_{R^{n}}
\end{array}\right], D \phi\right)_{R^{n}} d x \\
=|z|_{R^{n}}^{p-2} \int_{\Omega} \sum_{i=1}^{n}\left(\vec{a}_{i k}(x), z\right)_{R^{n}} \frac{\partial \phi}{\partial x_{i}} d x=|z|_{R^{n}}^{p-2} \int_{\Omega} \sum_{i=1}^{n} \sum_{j=1}^{n} a_{i j}^{k}(x) \frac{\partial \phi}{\partial x_{i}} z_{j} d x \\
=|z|_{R^{n}}^{p-2} \sum_{i=1}^{n} z_{j} \int_{\Omega}\left(\vec{a}_{i k}(x), D \phi\right)_{R^{n}} d x \\
=-|z|_{R^{n}}^{p-2} \sum_{j=1}^{n} z_{j}\left\langle\operatorname{div} \vec{a}_{j k}, \phi\right\rangle_{W^{-1, q}(\Omega) ; W_{0}^{1, p}(\Omega)}=J_{k},
\end{gathered}
$$

Then using (6.8), we get

$$
\begin{array}{r}
\lim _{k \rightarrow \infty} J_{k}=|z|_{R^{n}}^{p-2} \sum_{j=1}^{n} z_{j} \lim _{k \rightarrow \infty}\left\langle-d i v \vec{a}_{j k}, \phi\right\rangle_{W^{-1, q}(\Omega) ; W_{0}^{1, p}(\Omega)}= \\
=|z|_{R^{n}}^{p-2} \sum_{j=1}^{n} z_{j}\left\langle-d i v \vec{a}_{j 0}, \phi\right\rangle_{W^{-1, q}(\Omega) ; W_{0}^{1, p}(\Omega)}
\end{array}
$$

Making the converse transformations with (6.19) as we did it in (6.18), we come to the relation

$$
\lim _{k \rightarrow \infty}\left\langle-\operatorname{diva}\left(A_{k}, z\right), \phi\right\rangle_{W^{-1, q}(\Omega) ; W_{0}^{1, p}(\Omega)}=\left\langle-\operatorname{diva}\left(A_{0}, z\right), \phi\right\rangle_{W^{-1, q}}(\Omega) ; W_{0}^{1, p}(\Omega) .
$$

Since for every $i=1, \ldots n$ the sequences $\left\{d i v a_{i k}\right\}_{k \in N}$ are strongly convergent in $W^{-1, q}(\Omega)$, from $(6.18)-(6.20)$ it follows that

$$
\lim _{k \rightarrow \infty}\left\langle-\operatorname{diva}\left(A_{k}, z\right), \phi_{k}\right\rangle_{W^{-1, q}}(\Omega) ; W_{0}^{1, p}(\Omega)=\left\langle-\operatorname{diva}\left(A_{0}, z\right), \phi\right\rangle_{W^{-1, q}(\Omega) ; W_{0}^{1, p}}(\Omega)
$$


for each sequence $\left\{\phi_{k}\right\}_{k \in N} \subset C_{0}^{\infty}(\Omega)$ such that $\phi_{k} \stackrel{w}{\longrightarrow} \phi$ in $W_{0}^{1, p}(\Omega)$. Thus, summing up the above results, we obtain

$$
\left.\begin{array}{c}
\operatorname{diva}\left(A_{k}, z\right) \rightarrow \operatorname{div} a\left(A_{0}, z\right) \text { strongly in } W^{-1, q}(\Omega), \\
a\left(A_{k}, z\right)=A_{k}|z|_{R^{n}}^{p-2} \stackrel{w}{\longrightarrow} A_{0}|z|_{R^{n}}^{p-2} z \text { weakly }-* \text { in } L^{\infty}(\Omega) .
\end{array}\right\}
$$

As a result, combining properties (6.17) and (6.22), it has been shown that all suppositions of Lemma 6.3 are fulfilled. So, taking into account (6.9), (6.17), (6.22), and passing to the limit in inequality (6.16) as $k \rightarrow \infty$, we get

$$
\int_{\Omega} \phi(x)\left(\xi-a\left(A_{0}, z\right), D y_{0}-z\right)_{R^{n}} d x \geq 0, \quad \forall z \in R^{n}
$$

for all positive $\phi \in C_{0}^{\infty}(\Omega)$. After localization, we have

$$
\left(\xi-a\left(A_{0}, z\right), D y_{0}-z\right)_{R^{n}}, \quad \forall z \in R^{n}
$$

Since the operator $\mathfrak{\Im}_{1}$ is strictly monotone, it follows that

$$
\xi=a\left(A_{0}, D y_{0}\right)=A_{0}(x)\left|D y_{0}\right|_{R^{n}}^{p-2} D y_{0} .
$$

Therefore, equation (6.14) takes the form

$$
-\operatorname{div}\left(A_{0}(x)\left|D y_{0}\right|_{R^{n}}^{p-2} D y_{0}\right)+a_{0}\left|y_{0}\right|^{p-2}\left|y_{0}\right|=f \text { in } D^{\prime}(\Omega) \text {. }
$$

Hence the $\tau$-limit pair $\left(\mathrm{A}_{0}, \mathrm{y}_{0}\right)$ is an admissible to the problem (5.7)-(5.8), and this concludes the proof. $\square$

As an obvious consequence of this theorem (see (6.7), (6.9), (6.13), and (6.23)), we have the following result:

COROLLARY 6.6. Let $\left\{A_{\varepsilon}\right\}_{\varepsilon>0}$ be a sequence of matrices in $U_{\text {sol }} \subset M_{c}^{\beta}(\Omega)$. Let $A^{*} \in M_{\alpha}^{\beta}(\Omega)$ be its weak-* limit in $L^{\infty}\left(\Omega ; R^{n \times n}\right)$. Then for every $f \in W^{-1, q}(\Omega)$ the sequence $\left\{u_{\varepsilon}\right\}_{\varepsilon>0}$ of the solutions to the Dirichlet problems

$$
\left\{\begin{array}{c}
u_{\varepsilon} \in W_{0}^{1, p}(\Omega), \\
-\operatorname{div}\left(A_{\varepsilon}(x)\left|D u_{\varepsilon}\right|_{R^{n}}^{p-2} D u_{\varepsilon}\right)+a_{0}(x)\left|u_{\varepsilon}\right|^{p-2} u_{\varepsilon}=f \text { in } D^{\prime}(\Omega),
\end{array}\right.
$$

satisfies

$$
\begin{gathered}
u_{\varepsilon} \rightarrow \text { u weakly in } W_{0}^{1, p}(\Omega), \\
A_{\varepsilon}\left|D u_{\varepsilon}\right|_{R^{n}}^{p-2} D u_{\varepsilon} \rightarrow A^{*}|D u|_{R^{n}}^{p-2} D u \text { weakly in } L^{q}\left(\Omega, R^{n}\right),
\end{gathered}
$$

where $u$ is the solution to the problem

$$
\left\{\begin{array}{c}
u \in W_{0}^{1,2}(\Omega), \\
-\operatorname{div}\left(A^{*}(x)\left|D u_{\varepsilon}\right|_{R^{n}}^{p-2} D u\right)+a_{0}(x)|u|^{p-2} u=f \text { in } D^{\prime}(\Omega) .
\end{array}\right.
$$


Thus, in the case when $p=2$, we just come to the desired result: $H$-convergence of any sequence of matrices $\left\{A_{\varepsilon}\right\}_{\varepsilon>0} \subset U_{\text {sol }}$ is equivalent to its convergence with respect to the weak-* topology of $L^{\infty}\left(\Omega ; R^{n \times n}\right)$.

\section{References}

1 Bucur D., Buttazzo G., Variational Methodth in Shape Optimization Problems, Birkhauser, Boston: in Progress in Nonlinear Differential Equations and their Applications, volume 65, 2005 .

2 Bucur D., Trebeschi P., Shape optimization problem governed by nonlinear state equations, Proc. Roy. Soc. Edinburgh, Ser. A, 128(1998), 943-963.

3 C. Calvo-Jurado, J. Casado-Daz, Optimization by the homogenization method for nonlinear elliptic Dirichlet problems, Mediterranian J. of Mathematic, 4(2007), 53-63.

4 Dal Maso G., Murat F., Asymproric behaviour and correctors for Dirichlet problem in perforated domains with homogeneous monotone operators, Ann. Scoula Norm. Sup. Pisa Cl.Sci., no. 4, 24(1997), 239-290.

5 Gaevskii H., Greger K., Zaharias K., Nonlinear Operator Equations and Operator Differential Equations, Mir, Moscow, 1978(in Russian)

6 Lions J.-L., Some methods of Solving Non-Linear Boundary Value Problems, DunodGauthier-Villars, Paris, 1969.

7 Lurie K.A., Applied Optimal Control Theory of Distributed Systems, Plenum Press, NewYork, 1993

8 Murat F., Un contre-exemple pour le proleme de controle dans les coefficients, C.R.A.S.Paris, Ser. A 273(1971), 708-711.

9 Murat F., Teoremes de non-existence pour des problemes de controle dans le coeffcients, C.R.A.S. Paris, Ser. A 274(1972), 395-398.

10 Murat F., Tartar L., H-convergence, Topics in the mathematical modelling of composite materials, 21-43, Progr. Nonlinear Differential Equations Appl., Vol.31, Birkhauser, Boston, MA, 1997

11 Tartar L., Estimations fines de coefficients homogeneises, Ennio De Giorgi colloquium (Paris, 1983), 168-187, Res. Notes in Math., Vol.125, Pitman, Boston, MA, 1985.

12 Zgurovski M.Z., Mel'nik V.S., Nonlinear Analysis and Control of Physical Processes and Fields, Springer-Verlag, Berlin, 2004.

13 Zhikov V.V., Kozlov S.M., Oleinik O.A., Homogenization of Differential Operators and Integral Functionals, Springer Verlag, Berlin, 1994. 